\title{
Nondestructive Evaluation of Shoes Bonded Area Defect Composed of EVA Materials
}

\author{
S.-H. CHOI ${ }^{a}$ AND J.-Y. KIM ${ }^{b, *}$ \\ ${ }^{a}$ Department of Advanced Parts and Materials Engineering, Chosun University Graduate School \\ 375 Seosuk-dong, Dong-gu, Gwangju 501-750, South Korea \\ ${ }^{b}$ Department of Mechatronics Engineering, Chosun University, 375 Seosuk-dong, Dong-gu \\ Gwangju 501-750, South Korea
}

\begin{abstract}
The accurate detection of product defects in the assembly line is crucial to any industry. To achieve accurate detection, extensive studies have been conducted, in particular, on the nondestructive inspection technique. Shoe manufacturers, however, have yet to adopt a reliable defect-detection technique for their total inspection system. The deterioration of strength, one of the most common defects found in the shoe manufacturing process, is caused by the delamination in the bonding stage. Inadequate bonding between midsole and insole, the most serious of identified defects, has so far been detected only by cutting a sample shoe and examining it for its strength. For this study, the researcher tested the validity of some nondestructive techniques employed in inspecting shoes, particularly the infrared thermography technique and non contact air coupled ultrasonic testing, which are used to examine the bonded part of shoes made from ethylene vinyl acetate (EVA), the most common material used in manufacturing shoes. The study has thus confirmed the feasibility of applying a total inspection and nondestructive inspection technique to shoe inspection.
\end{abstract}

DOI: 10.12693/APhysPolA.123.333

PACS: $81.70 . \mathrm{Cv}$, 81.70.Fy, 81.05.Lg, 87.63.Hg

\section{Introduction}

The development of nondestructive inspection techniques has been remarkable in many industries, where defect inspection system plays a key role. Among such inspection systems, the infrared thermography technique examines the physical properties and defects of a sample without destroying it by measuring the infrared ray radiated through the sample. The technique, which is used mainly in the semiconductor, nuclear, defense, aerospace and automobile industries where an exceedingly high level of reliability and stability is required, is increasingly gaining significance with the advancement of relevant industries [1]. The non contact air-coupled ultrasonic testing, on the other hand, is used to inspect a sample with rough surfaces or those with very low or high temperature. It is also used when the conventional contact ultrasonic testing method cannot be employed due to the difficulty in applying a couplant in constricted spaces. The technique ensures reliable nondestructive inspection, as it compensates for the loss of energy triggered by the difference of sonic impedance between a sample and air by using a high-power ultrasonic pulser-receiver, a pre-amp and a high-sensitivity probe. The technique is used to detect the decay and defects of material with very high or low temperature, or to inspect curved surfaces of aircraft and the de-lamination of carbon composite material used for automobiles $[2,3]$. The study of a total inspection system employing these nondestructive inspection

*corresponding author techniques is growing in importance as shoe manufacturers have yet to establish an automated total inspection system.

The test equipment used in this study includes NAUT21, which is a non contact air coupled ultrasonic testing system, and P620, a thermography camera made by FLIR. Samples rubber/rubber and rubber/phylon (compressed EVA sponge) inflicted with artificial defects were tested using both the non contact air coupled ultrasonic test and infrared thermography probing. Here follows a brief introduction on the nondestructive inspection methodology and the feasibility of non contact air coupled ultrasonic probing and infrared thermography test, as well as the construction of the testing system.

\section{Composition of the testing equipment}

\subsection{Infrared thermography camera}

The P620 infrared thermography camera made by FLIR was used in this study. The camera measures the distribution of temperature on an object by detecting, among various wavelengths, infrared ray reflected from the object. Crucial here is the rate of radiation that varies according to the temperature, distance and property of the object. It is the slight differences in the radiation level that allow a tester to measure the temperature. The measurements of infrared ray reflected from an object are then calculated using the formula of the StefanBoltzmann law, which states a relationship between temperature and wavelength, before being converted into a graphical representation of the temperature distribution.

As shown in Fig. 1, the total standard radiant energy can be expressed as Eq. (1): 


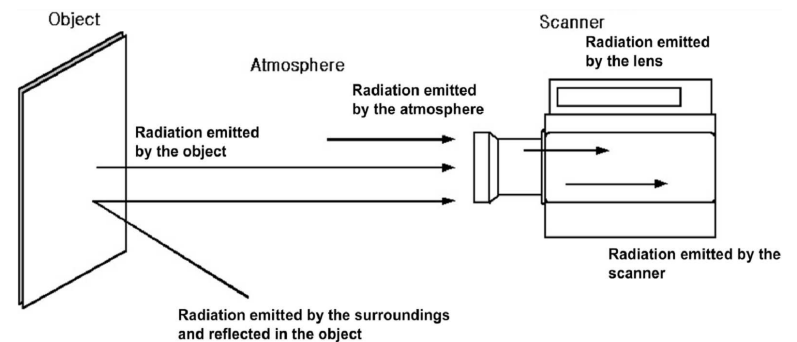

Fig. 1. Radiation contributions to the general measurement situation.

$$
\begin{aligned}
& I_{\mathrm{m}}=I\left(T_{\mathrm{obj}}\right) \times \tau \times \varepsilon+\tau(1-\varepsilon) \times I\left(T_{\mathrm{amb}}\right) \\
& \quad+(1-\tau) \times I\left(T_{\mathrm{afm}}\right) .
\end{aligned}
$$

The first term represents the radiation emitted by the surroundings and reflected in the object; the second term represents the radiation emitted by the object; and the third term represents the radiation emitted by the atmosphere.

\subsection{Non contact air coupled ultrasonic testing}

The non contact air coupled ultrasonic probe used in this study is composed of a high-sensitivity air probe, an ultra high-power ultrasonic PXI chassis, a coupled pulser-receiver, a pre-amp with a frequency filter and a high-speed digitizer, ensuring a high-speed processing of massive amounts of digital data [4]. It also consists of an automatic scanner attached with a probe, expanded hardware and computer installed with the LabVIEW operating software. The system is capable of various improvisations of system channeling, ranging from the reception of ultrasonic waves, triggering, control of the stage holding the test objects and transmission/reception of pulse signals, to data output. Figure 2 shows the construction of the non contact air coupled ultrasonic testing system.

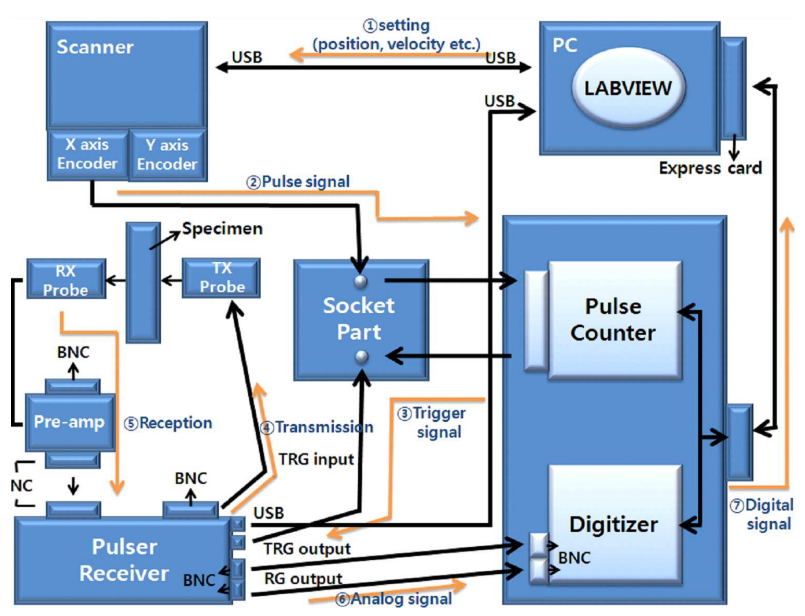

Fig. 2. Construction of the non contact air coupled ultrasonic testing system.

\section{Experimental methods and results}

\subsection{Production of the test specimen}

The EVA material used for shoe sponges, in particular, is lightweight, easy to form and inexpensive, unlike rubber and polyurethane. Such properties make it the most preferred choice for shock-absorbing substances used in shoes. Mainly used in the production of the shock-absorbing components of shoes called the "midsole" and the "insole", it is made by processing sponges that are cross-linked and foamed from EVA. The adhesive used in the bonded parts is hot-melt adhesive or HMA.

Samples for the detection of defects in the bonded parts of shoes by using a non contact air coupled ultrasonic testing and an infrared thermography testing were made as shown in Fig. 3. Sample 1 is a double-layered piece of standard shoe rubber and rubber bonded together, whereas sample 2 is a double-layered piece of rubber and EVA material, which is increasingly becoming popular in the industry. Sample 3 is a triple-layered piece of rubber and EVA material. The samples tested in the study consisted of at least one or two different materials bonded together, with each of them duplicated four times (two normal samples, two samples inflicted with artificial defects) totaling to 12 different samples.

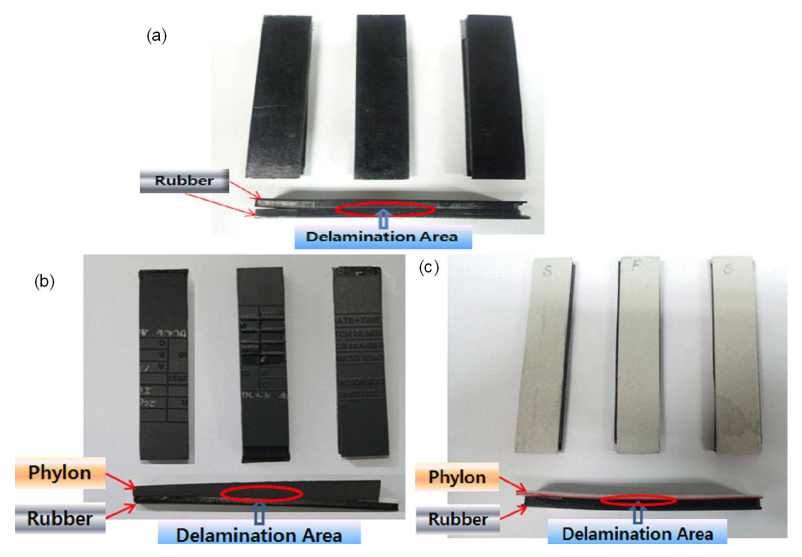

Fig. 3. Experiment specimens for NDT: (a) sample 1, (b) sample 2, (c) sample 3.

To prevent the ultrasonic waves from leaking on both ends of a sample or simply slipping through the seams when it goes through the boundary surface due to the samples' small size, thereby hindering the probing procedure, the seams of samples were sealed with corrugated cardboard and clay.

\subsection{Experimental methods}

3.2.1. Detection of defects using a non contact air coupled ultrasonic testing

Detection of defects in the bonded parts of a shoe using a non contact air coupled ultrasonic probing was conducted as follows: three sets of samples, each paired with normal and defective specimens, transmission probes used 0.4K20N R40 (AR1313), and receiving probes used 
0.4K20N-RX R40 (AR1101023). The pitch was set at $0.5 \mathrm{~mm}$, the transmission wave was set at 6 , and the gain value was set at $40.8 \mathrm{~dB}$. The scan speed was set at $100 \mathrm{~mm} / \mathrm{s}$, the transmission frequency was set at $380 \mathrm{kHz}$, and the transmission voltage was set at $300 \mathrm{~V}$. Experimental results were analyzed by averaging five scanned images.

\subsubsection{Detection of defects using the infrared} thermography technique

Active thermography has long been used in the field of nondestructive inspection. The simplest application of this is performed by subjecting a test sample or structure to heat. In this study, the transmission method [5], which is one of several infrared thermography techniques, was used employing a halogen lamp. To overcome the above limitation, the transmission method was used where heating is applied to the back side of the specimen. The heating source and the detector are located one on each side of the component to inspect. Generally the reflection approach is used for detection of discontinuities located close to the heated surface whereas the transmission approach allows detection of discontinuities close to the rear surface because of the spreading effect of the thermal front.

\subsection{Experimental results}

The following scanned images were obtained after applying a non contact air coupled ultrasonic probing technique to the three types of shoe samples. Interpretation of the images: if the penetration rate of the ultrasound wave is high, it appears as red in the scanned images, whereas defects appear as blue as the penetration rate drops in those spots.

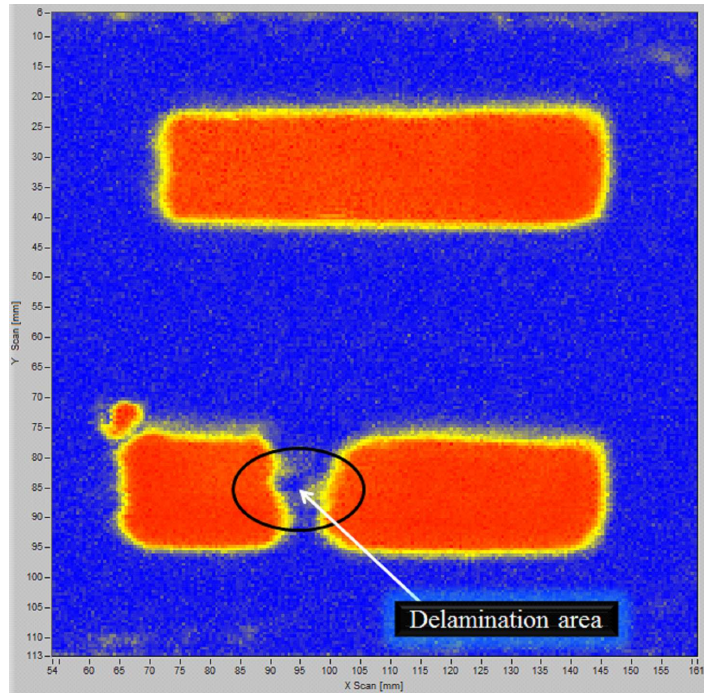

Fig. 4. C-scope image of sample 1.

In Fig. 4, which shows a scanned image of sample 1, the normal sample appears as red due to the high penetration of ultrasonic waves, whereas the other sample inflicted with artificial defects appears as blue as the penetration rate drops along the bonded surface where de-lamination has taken place.

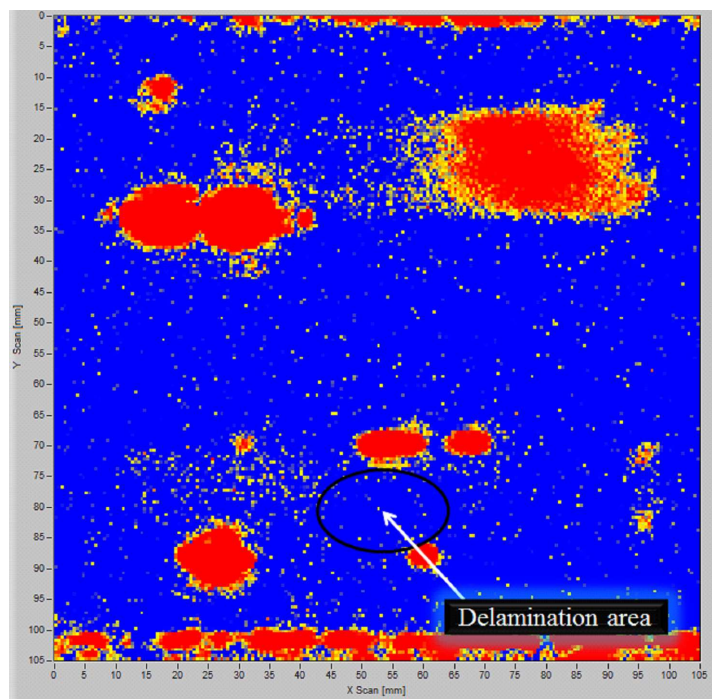

Fig. 5. C-scope image of sample 2.

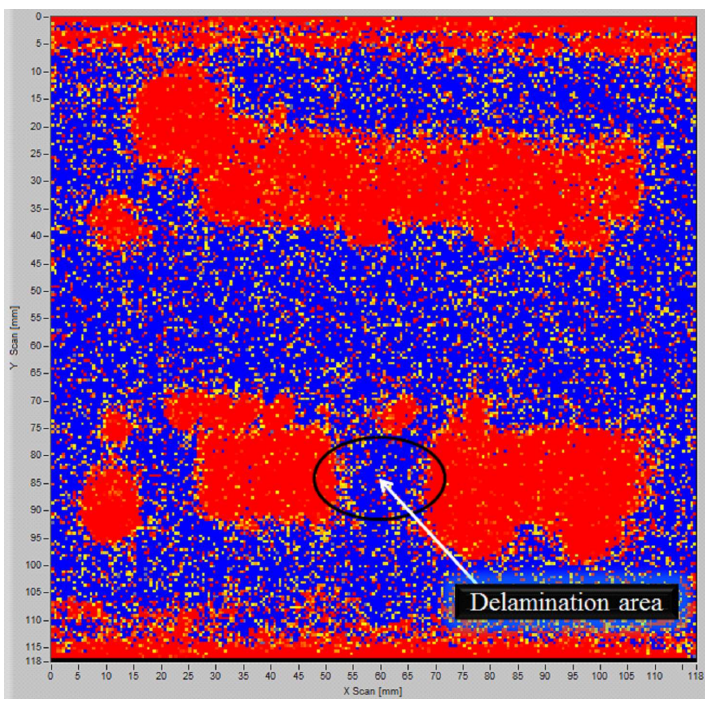

Fig. 6. C-scope image of sample 3.

In Figs. 5, 6, which show the scanned images of sample 2 and sample 3 , the test result is not satisfactory as the materials used in the midsole and the insole, respectively, significantly differed from each other, hence largely blocking the penetration of ultrasonic waves. This result may explain why the choice of probe in a non contact air couple ultrasonic probing technique is important. Further researches on this topic will be conducted in a subsequent study.

The following thermography images were obtained after applying an infrared thermography technique to three types of shoe samples. Interpretation of the images: the 


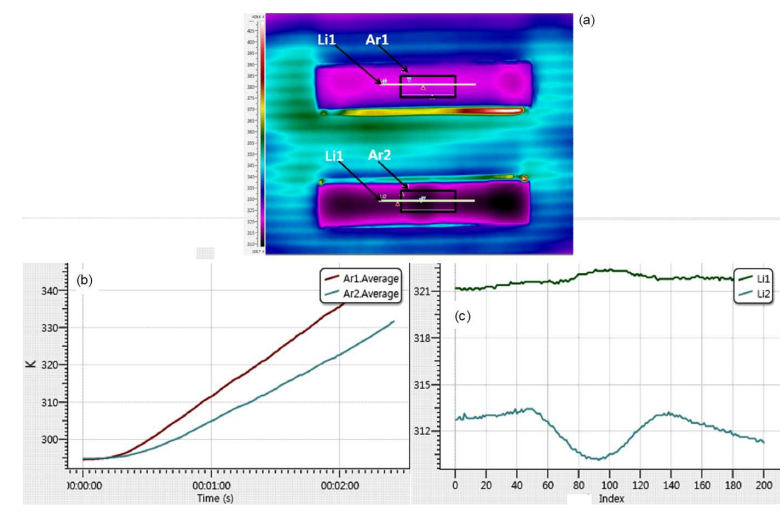

Fig. 7. Test result of sample 1: (a) thermography image, (b) average temperature over time, (c) temperature distribution along the line.

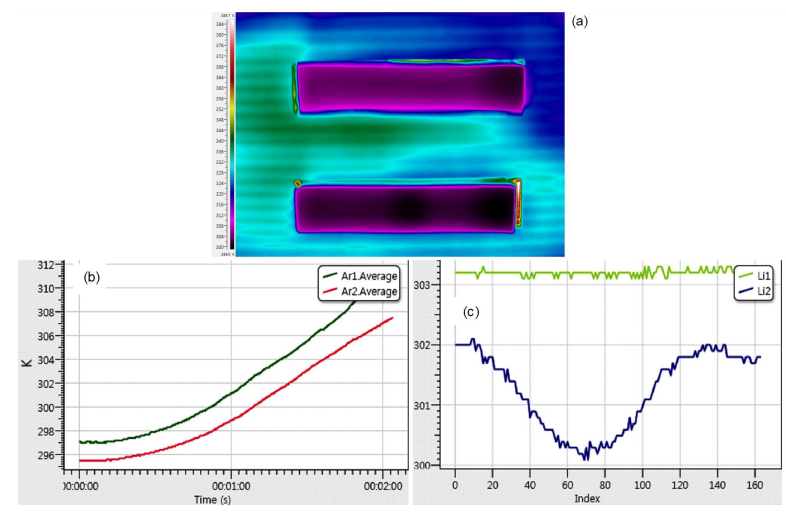

Fig. 8. As in Fig. 7, but for sample 2.

sample with no defects after three minutes of heating from a halogen lamp shows an even distribution of heat due to the constant conduction of heat to the sample, whereas the other sample inflicted with artificial defects shows cold spots in the defective areas as the heat conduction rate drops in those areas. Furthermore, to decrease errors in the temperature measurements, a rect-

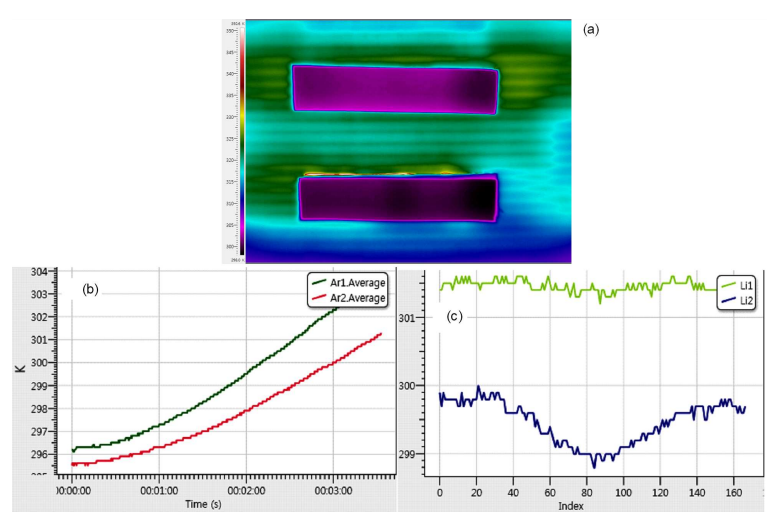

Fig. 9. As in Fig. 7, but for sample 3. angular area was specified at the center of each specimen and the average temperature in this area was measured and analyzed. Also one can draw a line measured temperature distribution of defects.

Figures 7-9 shows the thermography images of each sample, which helped accurately detect the defective areas.

\section{Conclusion}

This study's researcher has arrived at the following conclusions after conducting some nondestructive inspections on the bonded parts of shoes by using a non contact air coupled ultrasonic testing and an infrared thermography probing technique.

(1) A test employing a non contact air coupled ultrasonic probing technique shows that defects in shoes made from the same material was clearly detected, whereas detection was less satisfactory in the case of shoes made from more than two types of materials.

(2) Detection of defects in shoes made from more than two types of materials is believed to be possible if a different type of probe is employed.

(3) It was confirmed after an infrared thermography probing test on the three types of shoe samples that defects can be detected by examining the uneven distribution of heat conduction rate.

(4) This study's findings confirm that both the non contact air coupled ultrasonic and thermography probing technique can be effectively used in the field production line.

\section{Acknowledgments}

This research was financially supported by the Ministry of Education, Science Technology (MEST) and National Research Foundation of Korea (NRF) through the Human Resource Training Project for Regional Innovation.

\section{References}

[1] S.H. Choi, J.Y. Kim, Mater. Res. Innov. 15, 408 (2011).

[2] R. Kažys, A. Demčenko, E. Žukauskas, L. Mažeika, Ultrasonics 44, 819 (2006)

[3] V. Özkan, İ.H. Sarpün, Acta Phys. Pol. A 121, 184 (2012).

[4] S.N. Choi, H.N. Kim, H.J. Yoo, H.J. Cho, W.G. Hwang, J. Korean Soc. Nondestr. Testing 28, 331 (2008) (in Korean).

[5] X.P.V. Maldague, Infrared and Thermal Testing American Society for Nondestructive Testing, Vol. 3. 